\title{
Anaerobic carbon mineralization in hadal sediments
}

\author{
BO THAMDRUP ${ }^{1}$, CLEMENS SCHAUBERGER ${ }^{1}$, FRANK \\ WENZHÖFER ${ }^{1,2,3}$ AND RONNIE N GLUD ${ }^{1,4}$
}

${ }^{1}$ University of Southern Denmark

${ }^{2}$ Max Planck Institute for Marine Microbiology

${ }^{3}$ Alfred Wegener Institute Helmholtz Centre for Polar and Marine Research

${ }^{4}$ Tokyo University of Marine Science and Technology

Presenting Author: bot@biology.sdu.dk

Recent studies have demonstrated high oxygen respiration rates in the hadal seafloor compared to sediments on the adjacent abyssal plains. The high activity frequently results in the depletion of oxygen few centimetres below the hadal sediment surface, whereas typical oxygen penetration depths at abyssal sites are on the order of decimetres. The high hadal oxygen consumption rates result from focusing of relatively reactive organic matter at the trench bottom in combination with intermittent rapid burial through mass deposition events. In combination, the burial of reactive carbon and the shallow depth of oxygen depletion create favourable conditions for anaerobic carbon oxidation processes, which are otherwise mainly known from much shallower locations on continental margins. Little is known, however, about the occurrence and kinetics of anaerobic processes in hadal sediments, including the potential effect of extreme hydrostatic pressure on carbon mineralization.

Analyses of porewater and solid phase chemistry in sediments from the Kermadec and Atacama trenches demonstrated the sequential depletion with sediment depth of nitrate, reactive manganese, and poorly crystalline ferric iron, and the parallel accumulation of soluble manganese, ferrous iron and, eventually, iron sulfide. This classical redox zonation indicates the occurrence of denitrification, dissimilatory manganese and iron reduction, and sulfate reduction as terminal electron accepting processes coupled to carbon oxidation. In the Atacama Trench, sulfate reduction was further documented in shipboard incubations with ${ }^{35} \mathrm{SO}_{4}{ }^{2-}$, with rates peaking already at $10-20$ $\mathrm{cm}$ depth, and expressed in electron equivalents, the depthintegrated rates corresponded to up to $10 \%$ of oxygen respiration rates determined from the diffusive oxygen uptake. Thus, our results demonstrate that anaerobic processes can play a substantial role in the mineralization of organic matter in hadal sediments and that this involves the typical array of microbially catalysed redox processes known from shallower settings. The results raise questions concerning, e.g., the recruitment and composition of the anaerobic microbial communities involved as well as their overall diagenetic impact. 